\title{
Prospects for High Energy Light Isotope Measurements on Balloons
}

\author{
Scott P. Wakely* \\ University of Chicago, Chicago IL 60637 USA \\ E-mail: wakelyeuchicago.edu \\ S. Coutu ${ }^{1}$, D. Müller ${ }^{2}$, J. Musser ${ }^{3}$, S. Nutter ${ }^{4}$, N.H. Park ${ }^{2}$, M. Schubnell ${ }^{5}$, G. Tarlé ${ }^{5}$ \\ ${ }^{1}$ Pennsylvania State University, University Park, PA 16802 USA \\ ${ }^{2}$ University of Chicago, Chicago IL 60637 USA \\ ${ }^{3}$ Indiana University, Bloomington, IN 47405 USA \\ ${ }^{4}$ Northern Kentucky University, Highland Heights, KY 41099 USA \\ ${ }^{5}$ University of Michigan, Ann Arbor, MI 48109 USA
}

\begin{abstract}
A number of recent 'anomalies' detected in the cosmic-ray flux have underscored the importance of improving our understanding of cosmic-ray source and propagation processes. To this end, one of the key observational tasks is obtaining measurements of the relative abundances of the light cosmic-ray isotopes at relativistic energies (above $\sim 1 \mathrm{GeV} / \mathrm{n}$ ), where existing information is extremely scarce. In particular, measurements of the clock isotope ${ }^{10} \mathrm{Be}$ for a range of relativistic time dilations are urgently needed. However, such measurements present a severe experimental challenge. The required mass resolution can only be reached if magnetic spectrometers with strong magnetic fields are equipped with state-of-the-art high-resolution trackers, and combined with devices such as ring-imaging Cherenkov counters for precise velocity measurements. Additionally, large exposure factors are needed for good statistical accuracy. In this presentation, we will briefly review the goals and challenges of such measurements, and describe a new proposed instrument, HELIX (the High-Energy Light Isotope eXperiment), that is designed to meet these challenges on a long-duration balloon flight.
\end{abstract}

The 34th International Cosmic Ray Conference,

30 July- 6 August, 2015

The Hague, The Netherlands

${ }^{*}$ Speaker. 


\section{Introduction}

The past decade has witnessed a number of results in cosmic-ray astrophysics that seem to directly challenge much of our conventional understanding of the field. These results have been made possible by new balloon-borne and space-based missions that have pushed the boundaries in both energy reach and precision. At the same time, the sophistication of cosmic-ray data modeling has also increased, as simple analytic approximations are being displaced by comprehensive and ever more realistic computational models. Such models are of universal importance in connecting the characteristics of source particle populations to the observed particle fluxes at Earth. A well-constrained and comprehensive propagation model is, hence, a prerequisite for convincingly claiming that (for instance) a positron excess is due to a specific origin. A common theme found throughout the theoretical work is the importance of improving and extending the cosmic-ray measurements which most directly impact these models.

Elemental secondary-to-primary ratios such as Boron/Carbon, for instance, have very successfully been employed, with other data, to infer information on the nature and size of the cosmic-ray confinement region. These ratios, by themselves, however, are insufficient to fully constrain all model parameters. In the case of diffusion models with halos, for example, they are sensitive only to the ratio of the halo size to the diffusion coefficient, such that large degeneracies in the parameter space remain.

Isotopic abundance ratios, on the other hand, serve a complementary and perhaps even more important role in revealing the underlying astrophysical features of cosmic rays. They provide key tests of the "universality" of cosmic-ray propagation histories, by examining species with different Z/A ratios and overall abundance levels [1-3]. Furthermore, these ratios are not subject to modification by atomic properties such as condensation temperature or first ionization potential [4]. Most dramatic is the information on the time history of cosmic rays carried by radioactive clock isotopes, such as ${ }^{10} \mathrm{Be}$ (half-life of $1.39 \mathrm{Myr}$ ). Because Be is a pure secondary, the ratio of ${ }^{10} \mathrm{Be}$ to stable ${ }^{9} \mathrm{Be}$ is entirely determined by the propagation history of the cosmic rays. ${ }^{10} \mathrm{Be}$ has been famously used within the context of Leaky Box models, for example, to estimate a mean containment lifetime of $\sim 15 \mathrm{Myr}$ for cosmic rays in the Galaxy. Within diffusion halo models, the ${ }^{10} \mathrm{Be} /{ }^{9} \mathrm{Be}$ ratio can be used, together with constraints from the secondary/primary ratios, to estimate values for the diffusion coefficient and the halo size [5-7]. At energies of a few $\mathrm{GeV} / \mathrm{n}$, the ${ }^{10} \mathrm{Be} /{ }^{9} \mathrm{Be}$ can also provide strong discrimination between entire classes of propagation model $[6,8,9]$.

Despite their long-recognized importance, however, these measurements are not among the objectives of any current high-energy cosmic-ray mission. Nevertheless, while there are significant technical challenges to achieving such measurements, they are well within the capabilities of a modern balloon-borne instrument.

\section{Technique and Measurements}

At low energies (up to a few $100 \mathrm{MeV} / \mathrm{n}$ ) the $\mathrm{dE}$ vs $\mathrm{E}$ technique [10], which requires the cosmic-ray particle to deposit all of its energy in a layered detector, has been quite successful in producing isotopic measurements. With this technique, the ACE/CRIS space mission has now provided isotopic abundances with excellent statistics and mass resolution for all elements from $\mathrm{Li}$ 
$(Z=3)$ to $Z n(Z=30)$ [11-14]. However, many science questions call for measurements at higher energy, where the particles can no longer be absorbed in the detector volume. For instance, the clock ratio ${ }^{10} \mathrm{Be} /{ }^{9} \mathrm{Be}$ has been measured by ACE/CRIS only to about $\sim 200 \mathrm{MeV} / \mathrm{n}$, well below the region where the relativistic time dilation should lead to a characteristic increase of this ratio.

At higher energy, magnet spectrometers become the instrument of choice. With these, one measures the nuclear charge, $Z$, of cosmic-ray particles (typically through measurement of the specific energy loss by ionization), and one determines the magnetic rigidity $R=p c / Z e$ (where $p=$ $\beta \gamma m c$ is the momentum, $c$ the speed of light, and $Z e$ the electric charge) and the velocity $\beta$, in order to obtain the particle mass $m$. The rigidity is derived by tracking the particle through the magnetic field of the spectrometer, while the velocity is determined by a time-of-flight measurement (at lower energies), or from the signals of appropriately-chosen Cherenkov counters. As indicated by Equation 2.1 for the mass resolution $(\Delta m / m)$, the success of the measurement depends on excellent resolution for rigidity, $\Delta R / R$, and for velocity, $\Delta \beta / \beta$ :

$$
\left(\frac{\Delta m}{m}\right)^{2}=\left(\frac{\Delta R}{R}\right)^{2}+\gamma^{4}\left(\frac{\Delta \beta}{\beta}\right)^{2}
$$

The presence of the $\gamma^{4}$ term here makes the velocity measurement particularly challenging as energy increases. To clearly separate neighboring isotopes, one desires a mass resolution of at least $\sim 0.25$ mass units, corresponding to $\Delta m / m \sim 2.5 \%$ for ${ }^{10} \mathrm{Be}$. This is illustrated in Figure 1 , which shows simulated mass histograms for ${ }^{10} \mathrm{Be}$ and ${ }^{9} \mathrm{Be}$ with $2.5 \%$ and $10 \%$ mass resolutions, respectively. Clearly, the poorer resolution does not permit a successful measurement.

Recent magnet spectrometers used for isotopic measurements include the balloon instruments IMAX, BESS, BESS-Polar, and ISOMAX, and the space instruments AMS-01 and PAMELA. However, because of limitations in resolution or exposure, these experiments have produced isotopic composition data for only the lightest elements and at relatively low energies: AMS-01 and PAMELA for the elements $\mathrm{H}$ to B up to $1.4 \mathrm{GeV} / \mathrm{n}[15,16]$; IMAX for ${ }^{3} \mathrm{He} /{ }^{4} \mathrm{He}$ up to $4 \mathrm{GeV} / \mathrm{n}$ [17]; and ISOMAX, for the $\mathrm{Li}, \mathrm{Be}$, and $\mathrm{B}$ isotopes around 1-2 $\mathrm{GeV} / \mathrm{n}[8]$. We emphasize, in particular, that no high-statistics measurements of the ${ }^{10} \mathrm{Be} /{ }^{9} \mathrm{Be}$ ratio exist above a few hundred MeV/nuc (see Figure 4). 


\subsection{Rigidity Resolution and Multiple Coulomb Scattering}

The rigidity resolution, $\Delta R / R$, of a spectrometer is determined by two primary contributions. The first, a kinematic contribution, comes from the limited spatial resolution of the tracker used to measure the bending of the particle track in the magnetic field, $B$. The bending angle $\theta$ is, to first order, proportional to the inverse of the gyro-radius (i.e., proportional to $B / R$ ), and the resolution may therefore be characterized by a minimum detectable variation $\delta \theta \propto \delta(B / R) \propto\left(B / R^{2}\right) \delta R$. Consequently, the systematic limit in relative rigidity resolution, $\delta R / R$, is proportional to $(R / B) \delta \theta$, which increases linearly with the rigidity of the particle.

A second contribution to the rigidity resolution comes from multiple Coulomb scattering of the particle in the material of the tracking system. This leads to an uncertainty $\delta \theta$ in the measured bending angle, which to first order is inversely proportional to $R$. The corresponding contribution, $\delta R / R$, to the total rigidity resolution is a term proportional to $1 / B$, which depends on the composition and mass density (i.e., thickness in $X_{0}$ ) of the tracking system, but which is independent of $R$ or $Z$. Obviously, it is crucial that $B$ be as large as possible in order to minimize the multiple scattering contribution.

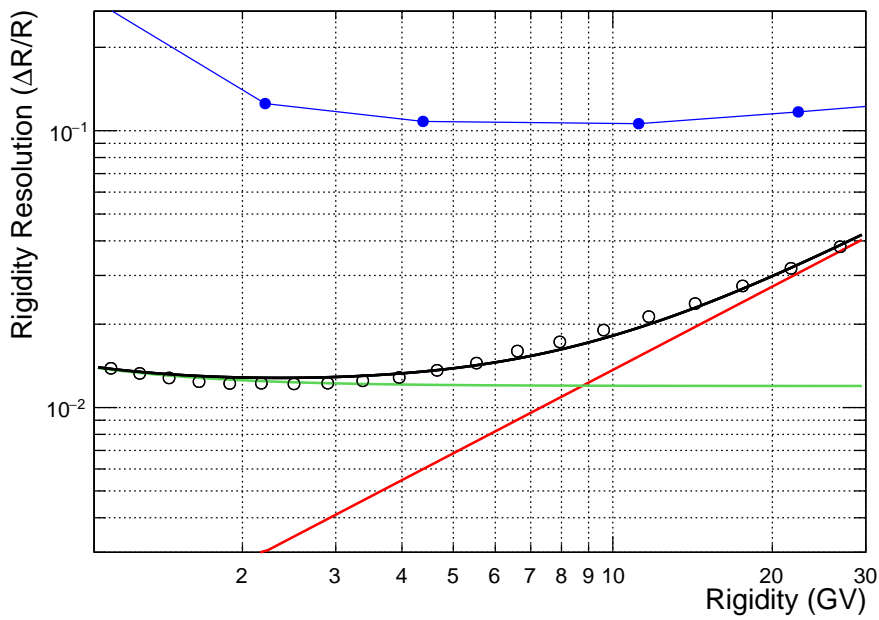

Figure 2: Monte Carlo simulations of rigidity resolution for vertical helium nuclei in the HELIX spectrometer. The open circles are simulation results, and the lines are a fit to the results: green is the multiple-scattering component, red is the kinematic component, and black is the quadrature sum of the two. The upper blue data points are the simulated response of the AMS- 02 detector, published by the AMS-02 collaboration [18].

The total rigidity resolution, $\Delta R / R$, consists of both the kinematic and scattering contributions added in quadrature, and is thus characterized by a nearly constant term (the 'resolution floor') at relatively low rigidity, and a linearly increasing term which dominates at high energy. When $\Delta R=R$, the spectrometer has reached its maximum detectable rigidity (MDR).

This behavior is demonstrated in Figure 2, which shows the simulated response of the balloonbased spectrometer described below. This instrument employs a $\sim 1 \mathrm{~T}$ magnetic field coupled with a thin, gas-based, tracking system. A resolution of under $2.5 \%$ is obtained up to $\sim 15 \mathrm{GV}$, which corresponds to $\sim 5 \mathrm{GeV} / \mathrm{n}$ for ${ }^{10} \mathrm{Be}$. This same level of performance cannot be achieved in a spectrometer of comparable matter thickness if the magnetic field is significantly lower. This is demonstrated with the blue markers in this figure, which are simulations of the AMS-02 detector performed by the AMS-02 collaboration [18]. Here, the $\sim 10 \%$ resolution is driven largely by the relatively low ( $\mathrm{B} \sim 0.15 \mathrm{~T}$ ) magnetic field of the permanent magnet. As shown in Figure 1, this resolution, even 
assuming perfect velocity determination, is insufficient to clearly separate ${ }^{10} \mathrm{Be}$ from ${ }^{9} \mathrm{Be}$ at all rigidities, even though the MDR of the instrument is quite large.

\subsection{Measurements of Particle Velocity}

In addition to rigidity, a measurement of the particle velocity is required to obtain a mass estimate. For $\Delta m / m$ in the range of $\sim 2-3 \%$, and assuming a spectrometer with $\Delta R / R \sim 2 \%$, this requires a weighted velocity resolution of $\gamma^{2} \Delta \beta / \beta \sim 2 \%$. Below $\sim 1 \mathrm{GeV} / \mathrm{n}$, time-of-flight (TOF) measurements can be employed, using the same scintillator paddles that provide charge determination. Absolute timing resolutions of $\sim 50 \mathrm{ps}$ can be achieved for $Z>3$ particles in systems of such paddles [8, 19]. This corresponds to resolutions $\Delta \beta / \beta \sim 7 \times 10^{-3}$ for $\sim 0.5 \mathrm{GeV} / \mathrm{n}$ particles over pathlengths of $\sim 2 \mathrm{~m}$. As energy is increased, however, the weighting term grows quickly and, at $\sim 1 \mathrm{GeV} / \mathrm{n}$, the resolution limit has typically been exceeded.

At higher energies, Cherenkov detectors must be used, but integrating counters also quickly become inadequate. Ring-Imaging Cherenkov (RICH) detectors, on the other hand, measure the Cherenkov angle independently of $\mathrm{Z}$, and are accurate even if only a few photons are detected. Thus, they provide the best performance and also cover a suitably large velocity range. In a simple proximity-focused RICH, the Cherenkov photons are directly collected on a detector plane with no special focusing elements. The best resolution is obtained with small index-of-refraction radiators (typically aerogels in the range $n=1.03-1.15$ ) and finely-pixelated photon detectors. There is a trade-off in $n$ to be considered: higher values will result in lower energy thresholds, but will produce larger Cherenkov rings, necessitating bigger, more expensive, focal planes.

To achieve the mass resolution needed for the ${ }^{10} \mathrm{Be} /{ }^{9} \mathrm{Be}$ measurement at $\sim 3 \mathrm{GeV} / \mathrm{n}$, a velocity resolution of $\Delta \beta / \beta \sim 1 \times 10^{-3}$ is required. Extensive simulations and results from previous instruments have shown that this can be achieved with an aerogel RICH with dimensions commensurate with the constraints of a balloon or space-based payload [20,21].

\section{HELIX Instrument}

The HELIX instrument is a proposed magnet spectrometer system configured specifically for the measurement of light isotopes. The design utilizes well-proven detector technology and benefits from the use of certain key components that already exist, such as silicon-strip detectors and the 1T superconducting magnet. As shown in Figure 3, the major components of the instrument, besides the magnet, include a hybrid gas/silicon particle tracker and an aerogel RICH. Scintillator paddles above the magnet bore and below the RICH form a time-of-flight system, provide the instrument trigger, and measure the particle charge. The HELIX instrument is designed to fly on a longduration balloon flight, for periods up to 14 days. This exposure, combined with the geometric acceptance of $0.1 \mathrm{~m}^{2} \mathrm{sr}$, is sufficient to enable high-statistics measurements of isotopic ratios even at high energy. A brief description of the main components follows:

Superconducting Magnet The magnetic field for HELIX is generated by a 2-coil superconducting magnet built by Cryomagnetics Incorporated, originally for the HEAT instrument [22]. It has been used in five successful balloon campaigns and provides an approximately uniform $1 \mathrm{~T}$ 
field within a rectangular warm bore measuring $51 \times 51 \times 61 \mathrm{~cm}^{3}$. To provide a pressurized environment for the tracking system, the bore will be sealed with a thin aluminum pressure cap which introduces less than $0.3 \mathrm{~g} \mathrm{~cm}^{-2}$ of material overburden. The original LHe capacity of the magnet is $260 \mathrm{~L}$, which provides a hold time of 7 days, at a mass of $420 \mathrm{~kg}$. This will be extended to 14 days using an external expansion Dewar being developed by the University of Michigan.

Hybrid Gas/Silicon Tracker To track particles through the field of the magnet, HELIX will utilize a hybrid gas/silicon tracking system containing a drift chamber tracker (DCT) sandwiched between 'outriggers' made from high-resolution silicon strip detectors. This combination affordably minimizes multiplescattering distortions by pairing a gas detector of intrinsically-low material thickness with high-performance silicon tracking layers on the outermost layers, where they do not contribute to the scattering budget. By using a 'cold', low-diffusion, drift gas such as $\mathrm{CO}_{2}$, a moderate drift field of $\sim 1 \mathrm{kV} / \mathrm{cm}$, and high-speed sampling readout electronics, a mean spatial resolution of $\sim 65 \mu \mathrm{m}$ can be achieved for $\mathrm{Z}>3$ particles over the appropriate drift distances [23,24].

To provide high-resolution measurements in the top and bottom layers of the tracker, we have acquired

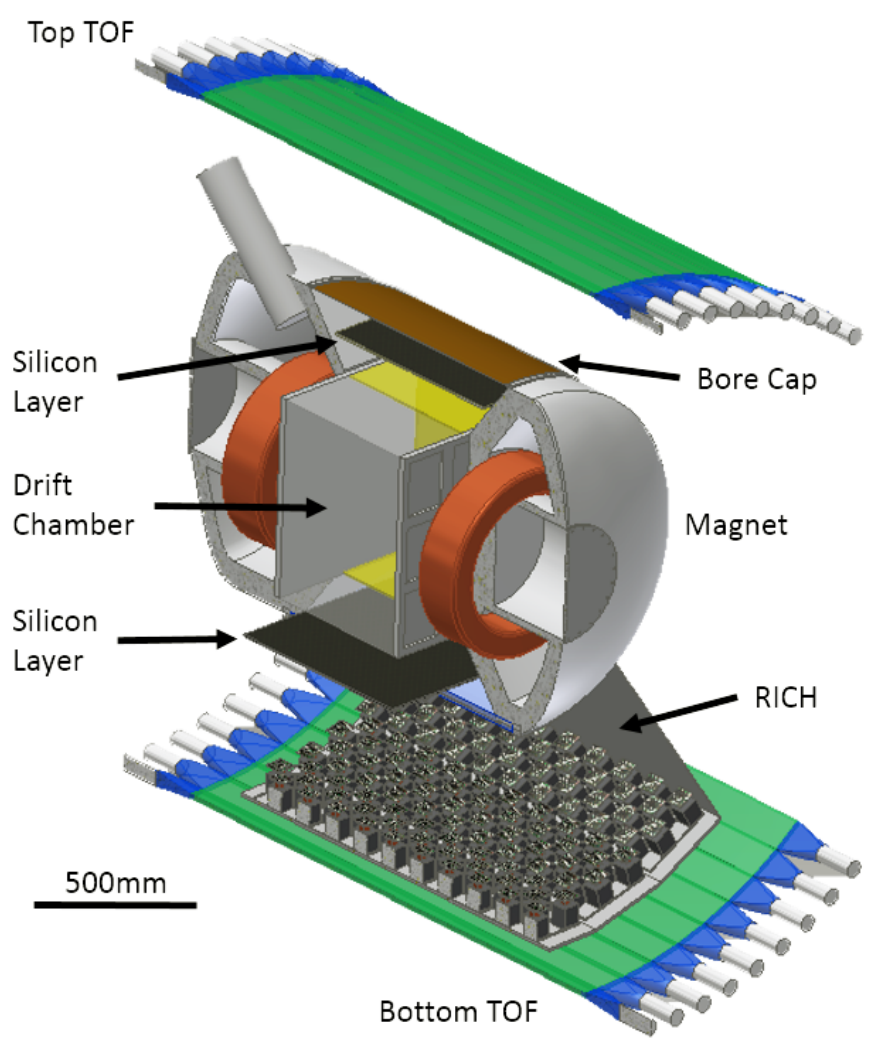

Figure 3: Partially-sectioned 3D model of the High Energy Light Isotope eXperiment detector systems, showing superconducting magnet, hybrid tracking system, two time-of-flight layers and ring-imaging Cherenkov detector.

600 silicon strip detectors (SSDs) originally designed for the Intermediate Silicon Layer (ISL) of the Fermilab/CDF silicon vertex detector [25]. The ISL SSDs are double-sided 300- $\mu$ m-thick sensors with a readout pitch of $112 \mu \mathrm{m}$ on both sides, and a stereo angle of $1.2^{\circ}$ for the back-side (n-type) strips. Using the simplest digital hit algorithms, this should be adequate for providing $\sim 32 \mu \mathrm{m}$ resolution in the bending plane of the magnet.

Time-of-Flight and Charge System The HELIX time-of-flight and charge system consists of two layers of $1.5 \mathrm{~cm}$-thick fast plastic scintillator paddles, located at the top and bottom of the instrumentation stack, with a total separation of $2.3 \mathrm{~m}$ (see Figure 3). To eliminate the need for heavy magnetic shielding or delicate field alignments, the paddles will be read out with SiPM devices. They will be coupled to a TDC system employing the TDC-GPX ASIC. Based on previous instruments and ongoing bench tests, we aim to achieve a time resolution of $<50 \mathrm{ps}$ for particles with $\mathrm{Z}>3$. 

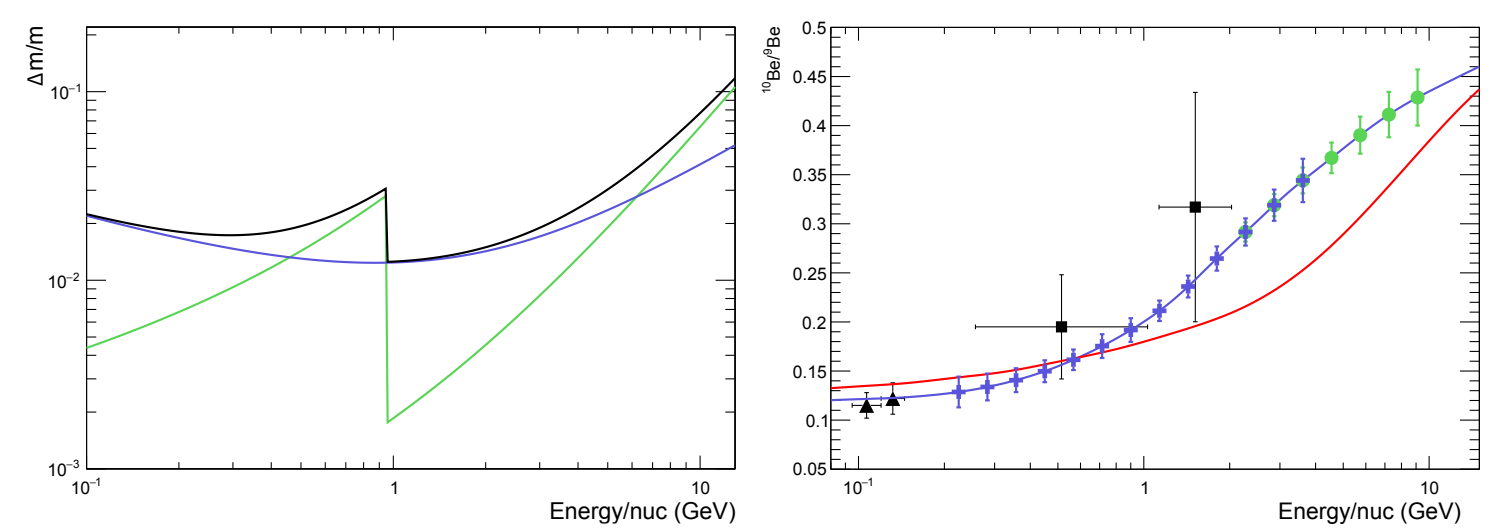

Figure 4: Left: Predicted relative mass resolution vs energy per nucleon for the HELIX instrument with ${ }^{10} \mathrm{Be}$ incident particles. The blue line is the contribution to the mass resolution from the spectrometer; the green line is the $\gamma^{2}$-weighted contribution from the velocity-determining instrument: the TOF below 1 $\mathrm{GeV} / \mathrm{n}$ and the RICH above $1 \mathrm{GeV} / \mathrm{n}$. The black line is the quadrature sum of the two. Right: Anticipated beryllium isotope ratio measured by HELIX. The blue crosses show results for a 14-day Antarctic flight with the instrument configuration described here. The green dots show results for a future 28-day exposure with an upgraded instrument. The blue line represents a Leaky Box model with a low-density Local Bubble and the red line represents a diffusion halo model with reacceleration [8]. Also shown on the plot are the results of ACE/CRIS (triangles) and ISOMAX (squares).

Ring Imaging Cherenkov Detector For measurements of particle velocity at high energy, HELIX will use a proximity-focused aerogel RICH detector. On the basis of extensive GEANT4 Monte Carlo simulations, we adopt the following configuration: aerogel radiator with $n=1.15$ (threshold energy $\sim 1 \mathrm{GeV} / \mathrm{n}$ ), thickness $10 \mathrm{~mm}$, expansion length $500 \mathrm{~mm}$, and pixel size $6 x 6 \mathrm{~mm}^{2}$. The $1.32 \mathrm{~m}^{2}$ focal plane will consist of an array of multi-channel SiPM devices. The pixels will be cooled using a thermo-electric design adapted from atmospheric Cherenkov telescope cameras [26]. To reduce costs, the image plane will be partially populated with sensors, arranged in a checkerboard pattern. This lowers the velocity resolution of the device, but still enables measurements of the ${ }^{10} \mathrm{Be} /{ }^{9} \mathrm{Be}$ up to about $\sim 4 \mathrm{GeV} / \mathrm{n}$. In a future upgrade of the instrument, we will complete the focal plane and introduce refinements of the radiator design to further improve the velocity resolution [27].

\subsection{Expected Performance and Results}

HELIX is designed to allow for $4 \sigma$ mass separation for adjacent light isotope peaks, which corresponds to $0.25 \mathrm{amu}$. Figure 4 (left panel) shows the expected HELIX relative mass resolution $(\Delta m / m)$ versus kinetic energy per nucleon for ${ }^{10} \mathrm{Be}$ nuclei. This plot is based on full Monte Carlo simulations and uses the instrument configuration described above. Due to the thin tracker and large magnetic field, excellent mass resolution is obtained from a few hundred $\mathrm{MeV} / \mathrm{n}$, where the results can be compared with the ACE/CRIS measurement, all the way to $\sim 4 \mathrm{GeV} / \mathrm{n}$, well beyond the low-statistics measurement by ISOMAX at 1.1-2.0 GeV/n. With future upgrades to the tracker and $\mathrm{RICH}$, this sensitivity range can eventually be extended to $\sim 10 \mathrm{GeV} / \mathrm{n}$.

The right side of Figure 4 shows the expected HELIX results on the ratio of ${ }^{10} \mathrm{Be}$ to ${ }^{9} \mathrm{Be}$. In addition to results from the 14-day flight discussed here, a future 28-day exposure in an upgraded 
configuration is also shown. Clearly, even the initial HELIX results will provide very strong constraints on the behavior of the ${ }^{10} \mathrm{Be} /{ }^{9} \mathrm{Be}$ ratio, reaching with good statistics well into the region where time dilation significantly affects the radioactive isotope lifetime. From this 14-day flight alone, more than $1500{ }^{10} \mathrm{Be}$ nuclei are expected above $2 \mathrm{GeV} / \mathrm{n}$, an area where no measurements currently exist.

Other Measurements In addition to the ${ }^{10} \mathrm{Be} /{ }^{9} \mathrm{Be}$ ratio, HELIX will measure several other important isotopic abundance ratios, including the ${ }^{3} \mathrm{He} /{ }^{4} \mathrm{He}$ abundance ratio. Because the relative mass difference between these isotopes is large, HELIX has good mass separation from $200 \mathrm{MeV} / \mathrm{n}$ to beyond $\sim 8 \mathrm{GeV} / \mathrm{n}$. HELIX measurements will overlap on the low-energy side with the recent high-statistics results from PAMELA, and extend to energies where no direct measurements currently exist. HELIX will also explore, for the first time, the ${ }^{22} \mathrm{Ne} /{ }^{20} \mathrm{Ne}$ ratio at energies above a few hundred $\mathrm{MeV} / \mathrm{n}$. Other isotopic and elemental measurements, such as ${ }^{7} \mathrm{Li} /{ }^{6} \mathrm{Li},{ }^{10} \mathrm{~B} /{ }^{11} \mathrm{~B}, \mathrm{~B} / \mathrm{C}$, and others will also be obtained with good statistics to high energy.

\section{References}

[1] Coste, B., Derome, L., Maurin, D., \& Putze, A. A\&A 539 (Mar., 2012) A88, [arXiv:1108.4349].

[2] Tomassetti, N. Ap\&SS 342 (Nov., 2012) 131-136, [arXiv:1210 . 7355].

[3] Webber, W. R. Advances in Space Research 19 (May, 1997) 755-758.

[4] Prantzos, N. A\&A 542 (June, 2012) A67, [arXiv:1203.5662].

[5] Simpson, J. A., \& Garcia-Munoz, M. Space Sci. Rev. 46 (1988) 205-224.

[6] Simon, M. vol. 4 of Proc. 26th ICRC (Salt Lake), p. 211, Aug., 1999.

[7] Strong, A. W., Moskalenko, I. V., \& Ptuskin, V. S. Annual Review of Nuclear and Particle Science 57 (Nov., 2007) 285-327, [astro-ph/0701517].

[8] Hams, T., Barbier, L. M., Bremerich, M., et al. ApJ 611 (Aug., 2004) 892-905.

[9] Putze, A., Derome, L., \& Maurin, D. A\&A 516 (June, 2010) A66, [arXiv:1001.0551].

[10] Stone, E. C., Frandsen, A. M., Mewaldt, R. A., et al. Space Sci. Rev. 86 (July, 1998) 1-22.

[11] Wiedenbeck, M. E., Binns, W. R., Christian, E. R., et al. ApJ 523 (Sept., 1999) L61-L64.

[12] Wiedenbeck, M. E., Yanasak, N. E., Cummings, A. C., et al. Space Sci. Rev. 99 (Oct., 2001) 15-26.

[13] Yanasak, N. E., Wiedenbeck, M. E., Mewaldt, R. A., et al. ApJ 563 (Dec., 2001) 768-792.

[14] de Nolfo, G. A., Moskalenko, I. V., Binns, W. R., et al. Advances in Space Research 38 (Jan., 2006) 1558-1564, [astro-ph/0611301].

[15] Aguilar, M., Alcaraz, J., Allaby, J., et al. ApJ 736 (Aug., 2011) 105, [arXiv: 1106.2269 ].

[16] Menn, W., Bogomolov, E. A., Y Krut'kov, S., et al. Journal of Physics Conference Series 409 (Feb., 2013) 012030.

[17] Reimer, O., Menn, W., Hof, M., et al. ApJ 496 (Mar., 1998) 490.

[18] Ambrosi, G., et al. Proc. 33rd ICRC (Rio), 2013.

[19] Ahlen, S. P., Greene, N. R., Loomba, D., et al. ApJ 534 (May, 2000) 757-769.

[20] Pereira, R. in Proceedings of Como 2007, pp. 901-905, June, 2008. arXiv: 0801.3250.

[21] Giovacchini, F., et al. NIM A 766 (Dec., 2014) 57-60.

[22] Barwick, S. W., Beatty, J. J., Bower, C. R., et al. NIM A 400 (Feb., 1997) 34-52.

[23] Bock, P., Heintze, J., Kunst, T., Schmitt, B., \& Smolik, L. NIM A 242 (1986) 237.

[24] Haino, S. No. 8 in MAPS: Methods of Analysis for Physics in Space, 2009.

[25] Aaltonen, T., Behari, S., Boveia, A., et al. NIM A 729 (Nov., 2013) 153-181, [arXiv: 1301. 3180].

[26] Meagher, K. J. vol. 9145 of SPIE Conference Series, p. 33, July. arXiv:1407. 3271.

[27] Barnyakov, A. Y., Barnyakov, M. Y., Bobrovnikov, V. S., et al. NIM A 553 (Nov., 2005) 70-75. 